\title{
Editorial: Lactic Acid Bacteria Within the Food Industry: What Is New on Their Technological and Functional Role
}

\author{
Paola Lavermicocca ${ }^{1}$, Cristina Reguant ${ }^{2}$ and Joaquin Bautista-Gallego ${ }^{3 *}$ \\ ${ }^{1}$ Institute of Sciences of Food Production, National Council of Research of Italy, Bari, Italy, ${ }^{2}$ Departament de Bioquímica i \\ Biotecnologia, Facultat d'Enologia, Universitat Rovira i Virgili, Tarragona, Spain, ${ }^{3}$ Departamento de Ciencias Biomédicas \\ (Área de Microbiología), Facultad de Ciencias, Universidad de Extremadura, Badajoz, Spain
}

Keywords: fermentation processes, product preservation, food quality, food safety, health promoting features

\section{Editorial on the Research Topic}

Lactic Acid Bacteria Within the Food Industry: What Is New on Their Technological and Functional Role

\section{OPEN ACCESS}

Edited by:

Eugenia Bezirtzoglou,

Democritus University of

Thrace, Greece

Reviewed by:

Svetoslav Todorov,

University of São Paulo, Brazil

Yiannis Kourkoutas,

Democritus University of

Thrace, Greece

*Correspondence:

Joaquin Bautista-Gallego

joaquin.bautistagallego@gmail.com

Specialty section:

This article was submitted to

Food Microbiology,

a section of the journa

Frontiers in Microbiology

Received: 17 May 2021

Accepted: 21 June 2021

Published: 09 July 2021

Citation:

Lavermicocca $P$, Reguant $C$ and Bautista-Gallego J (2021) Editorial: Lactic Acid Bacteria Within the Food

Industry: What Is New on Their Technological and Functional Role.

Front. Microbiol. 12:711013. doi: 10.3389/fmicb.2021.711013

\section{INTRODUCTION}

The application of lactic acid bacteria (LAB) in food processing has a very long history (Leroy and De Vuyst, 2004), however the research continuously provides new insight. The biotransformation performed by LAB is implemented in many industrial processes since selected pro-technological LAB - through their lactic acid fermentative activity - can start and/or modulate the fermentation by producing acidic metabolites and other biomolecules such as enzymes, antimicrobials, and molecules contributing to aroma and texture. Through their proteolytic activity, LAB are able to degrade proteins into small peptides and free amino acids converted, in turn, to various metabolites-alcohols, aldehydes, acids, and ester compounds. Furthermore, lipolysis, glycolysis, and pyruvate metabolism performed by LAB produce many metabolites including aromatic compounds acting as flavor compounds (Leroy and De Vuyst, 2004). All these molecules contribute to the overall quality, shelf-life, and safety of foods, by enhancing their technological, sensorial, nutritional and functional features (Ravyts et al., 2012). It is worthy to note that the functional relevance of biotransformation operated by $\mathrm{LAB}$ relies also to their ability in modifying the bioavailability of bioactive molecules composing the food (Debelo et al., 2020). To successfully manage food processing and provide consumers with healthy foods, the genetic traits, and metabolisms of LAB have been deeply investigated in order to select strains suitable for specific applications (Giraffa, 2014).

The most common food-related LAB include species of the genera Lactobacillus, Lactococcus, Leuconostoc, Enterococcus, Pediococcus, Streptococcus, and Weissella. Within the phylum Firmicutes, the majority of LAB belongs to the order of Lactobacillales. Recently, the genus Lactobacillus has been reclassified into 25 genera (Zheng et al., 2020). However, to be applied in food processing LAB species must be recognized as safe (GRAS) by Food and Drug Administration (FDA) or must have achieved the Qualified Presumption of Safety (QPS) status by European Food Safety Authority (EFSA) (EFSA Panel on Biological Hazards et al., 2017).

Foods represent also the main source of LAB strains having probiotic features. Besides, food components play a role as vector in delivering probiotic populations by protecting them through the gastro-intestinal tract and by sustaining their gut colonization (Flach et al., 2018). 
Probiotic bacteria-defined as "live microorganisms that, when administered in adequate amounts, confer a health benefit on the host" (Hill et al., 2014) - mainly belonging to Lactobacillus and Bifidobacterium genera, are widely used in commercial products, particularly in milk-based preparations, that fall in the category of functional foods. Gut colonization by individual strains selected for beneficial functions strengthen the barrier function played by the epithelial and endothelial gut cells. Furthermore, their implantation modifies the resident microbial populations favoring the increase of those beneficial while limiting potential pathogens and, by modulating the components of the intestinal immune system, may stimulate the host immune response (Chugh and Kamal-Eldin, 2020). In the last decades, consumer's and market's interest for foods characterized by functional attributes has determined the widespread of research on probiotic foods. Particular interest is devoted to vegetable and fruit matrices rich in bioactive molecules that may act as carrier for probiotic microorganisms, producing innovative symbiotic (Flach et al., 2018).

Furthermore, the research and commercial interest in selecting strains for specific food processing, suggest the need for an update on the ongoing studies regarding metabolisms, genetic traits, fermentation performances, and functional features of LAB. Thus, the information obtained in this Research Topic can provide the food industry with rigorous scientific demonstrations for even more efficient biotransformation processes.

The Research Topic "Lactic Acid Bacteria within the Food Industry: What is New on their Technological and Functional Role" belongs to the Food Microbiology section in the Frontiers in Microbiology journal. It covers a total of 12 contributions divided in two reviews and 10 original research papers. Many of the most relevant researchers in the field have collaborated in its elaboration.

We present an overview of these papers which can be grouped under different research themes as follows: (i) probiotic and health-promoting characteristics for the production of functional foods and beverages; (ii) Interactions with other microorganisms; (iii) new technological approaches. The diversity of research displayed in this Research Topic demonstrates the important potential of these microorganisms and its impact in the food industry.

In the first group of papers, Hernández-Alcántara et al. have evaluated different probiotic properties of $L$. plantarum strains, such as their survival in the human digestive tract and in vivo studies using a murine model. Two strains (M5MA1B2 and M9MG6-B2) were able to tolerate the gastrointestinal stresses and displayed in vitro high adhesion capacity to Caco2 cells. Furthermore, they showed great biofilm formation characteristics which indicate a potential capability for intestine colonization, and an improvement of the surface area of the intestinal epithelium in the in vivo murine model. A similar study was carried out by Baliyan et al. who isolated potential probiotics microorganisms from a cereal-based traditional fermented beverage -lugri- (rice, wheat and barley) focusing in the functional and safety characteristics of this kind of beverage. Lacticaseibacillus paracasei LUL:01 exhibited the best performance and potential for its application in functional food formulation (milk-based formulation).

In addition, Cordeiro et al. have described the therapeutic effect in an induced colitis mice model of a Minas Frescal cheese made using the probiotic bacteria L. lactis NCDO 2118. They showed that this microorganism was able to limit the histopathological damages and restore intestinal barrier by increasing expression of gene related to tight junction protein and modulated cytokine production in mice. Furthermore, this functional cheese was able to produce high levels of bioactive peptides with antihypertensive, antioxidant, and antidiabetic activities.

Verni et al. studied the use of brewers' spent grain, a byproduct of the brewing industry, as a source to increase the intake of antioxidant compounds. To enable the release or synthesis of these compounds, a fermentation with different selected Lactiplantibacillus plantarum strains and treatment with a commercial xylanase was carried out.

Cataldo et al. evaluated the production of gammaaminobutyric acid through fermentation of strawberry and blueberry juices by Levilactobacillus brevis CRL 2013. A significantly higher production was reached in the case of fermented strawberry juice and it was able to modulate the expression of $\operatorname{cox}-2$ in lipopolysaccharide stimulated macrophages and exerted a remarkable anti-inflammatory effect. This study supports the potential use of this kind of fermented juice to reduce the inflammatory response of chronic inflammatory diseases.

Regarding to the interactions of LAB with other microorganisms, Medved'ová et al. have studied the effect of different combinations of LAB on the growth of different Staphylococcus aureus and Escherichia coli strains during the ripening of curd cheeses. This study emphasizes the importance of the use of LAB starter cultures to improve the sensory profile and safety conditions of the final product. In the same trend, Canon et al. reviewed the different ways available at present that could be used to create positive interactions between LAB: different types of positive interactions; co-cultivation and their mechanisms to reach the positive interactions; possible strategies that could be used to assemble LAB; and the particular role of nutritional dependencies.

With concern of the new technological approaches for LAB, four original articles and one review overview different studies to their implementation. Speranza et al. have studied the optimization of a fish fermented product using two L. plantarum strains. Both strains reduced the fermentation time and ensured good microbiological, chemico-physical, and sensorial quality of the final product.

Liu et al. have provided an approach to investigate the molecular mechanisms of formation and metabolic pathways of flavors in rice-acid fermented with $L$. paracasei H411 at different time and inoculation (co-inoculation with Kluyveromyces marxianus) methods. At transcriptional level, they detected that the genes related to amino sugar and nucleotide sugar metabolism and starch and sucrose metabolism affected the energy required for the growth of $L$. paracasei in the early stage. Even more, a different expression of those genes 
was detected in the growth of $L$. paracasei in the presence of K. marxianus.

Valerio et al. delved in the selection of LAB strains able to produce EPS in liquid sourdoughs based on pseudocereal flours and evaluated the effect of its composition on EPS production and protein degradation. Thus, the modulation of flour type, DY and sucrose content can stimulate the metabolic activities of Weisella cibaria and L. plantarum modulate the fermentation process, enriched in the EPS content. Furthermore, this increment in the EPS production was detected in the presence of pseudocereals amaranth or quinoa.

Kazou et al. compared the bacterial (and yeast/fungal) microbiota of different Greek kefir samples, using classical microbiological and amplicon-based metagenomics approaches. They identified mainly Lentilactobacillus kefiri, Leuconostoc mesenteroides, Lacticaseibacillus rhamnosus, Streptococcus thermophilus, Lactococcus lactis, and Leuconostoc mesenteroides. Yeasts were also isolated and identified during kefir production, being K. marxianus, Debaryomyces hansenii, and Saccharomyces cerevisiae the mostly identified yeast species. Even more, some (opportunistic) pathogens were detected at home-made kefir samples, which indicates poor hygiene practices.

Finally, Virdis et al. have reviewed the role of lactic acid bacteria in wine, focusing on the malolactic fermentation.

\section{REFERENCES}

Chugh, B., and Kamal-Eldin, A. (2020). Bioactive compounds produced by probiotics in food products. Curr. Opin. Food Sci. 32, 76-82. doi: $10.1016 /$ j.cofs.2020.02.003

Debelo, H., Li, M., and Ferruzzi, M. G. (2020). Processing influences on food polyphenol profiles and biological activity. Curr. Opin. Food Sci. 32, 90-102. doi: $10.1016 /$ j.cofs.2020.03.001

EFSA Panel on Biological Hazards, Ricci, A., Allende, A., Bolton, D., Chemaly, M., Davies, R., et al. (2017). Scientific opinion on the update of the list of QPSrecommended biological agents intentionally added to food or feed as notified to EFSA. EFSA J. 15:e04664. doi: 10.2903/j.efsa.2017.4664

Flach, J., van der Waal, M. B., van den Nieuwboer, M., Claassen, E., and Larsen, O. F. A. (2018). The underexposed role of food matrices in probiotic products: reviewing the relationship between carrier matrices and product parameters. Crit. Rev. Food Sci. Nutr. 58, 2570-2584. doi: 10.1080/10408398.2017.1334624

Giraffa, G. (2014). "Overview of the ecology and biodiversity of the LAB," in Lactic Acid Bacteria: Biodiversity and Taxonomy, Chapter 4, eds W. H. Holzapfel and B. J. Wood (West Sussex: John Wiley \& Sons), 45-54.

Hill, C., Guarner, F., Reid, G., Gibson, G. R., Merenstein, D. J., Pot, B., et al. (2014). The International Scientific Association for probiotics and prebiotics consensus statement on the scope and appropriate use of the term probiotic. Nat. Rev. Gastroenterol. Hepatol. 11, 506-514. doi: 10.1038/nrgastro.2014.66
Furthermore, they delved in the study of: (i) their contribution to the sensorial profile (citrate and glycosidase metabolism); (ii) impact on wine color (reduction of anthocyanin glucosides); (iii) production of volatile thiols; (iv) other diverse activities (reduction of the use of bentonite); and their negative effects (biogenic amines, ethyl carbamate).

The varied contributions to this Research Topic are evidence of the study undertaken by researchers that provide an updated and high-quality overview of the impact of lactic acid bacteria and their future perspectives. We hope that this Research Topic informs readers properly about the benefit of this product and the challenges that have yet to be overcome in this field.

\section{AUTHOR CONTRIBUTIONS}

All authors listed have made a substantial, direct and intellectual contribution to the work, and approved it for publication.

\section{ACKNOWLEDGMENTS}

We would like to thank all the contributing authors for their interest in this Research Topic. A special thank must go to all reviewers that devoted their precious time to the realization of this Research Topic.
Leroy, F., and De Vuyst, L. (2004). Lactic acid bacteria as functional starter cultures for the food fermentation industry. Trends Food Sci. Technol. 15, 67-78. doi: 10.1016/j.tifs.2003. 09.004

Ravyts, F., De Vuyst, L., and Leroy, F. (2012). Bacterial diversity and functionalities in food fermentations. Eng. Life Sci. 12, 356-367. doi: 10.1002/elsc.201100119

Zheng, J., Wittouck, S., Salvetti, E., Franz, C. M., Harris, H. M., Mattarelli, P., et al. (2020). A taxonomic note on the genus Lactobacillus: description of 23 novel genera, emended description of the genus Lactobacillus Beijerinck 1901, and union of Lactobacillaceae and Leuconostocaceae. Int. J. Syst. Evol. Microbiol. 70, 2782-2858. doi: 10.1099/ijsem.0.004107

Conflict of Interest: The authors declare that the research was conducted in the absence of any commercial or financial relationships that could be construed as a potential conflict of interest.

Copyright (c) 2021 Lavermicocca, Reguant and Bautista-Gallego. This is an openaccess article distributed under the terms of the Creative Commons Attribution License (CC BY). The use, distribution or reproduction in other forums is permitted, provided the original author(s) and the copyright owner(s) are credited and that the original publication in this journal is cited, in accordance with accepted academic practice. No use, distribution or reproduction is permitted which does not comply with these terms. 